Review

\title{
Impacts of Large Scale Wind Penetration on Energy Supply Industry
}

\author{
John Kabouris and Fotis D. Kanellos * \\ Hellenic Transmission System Operator, System Planning Department, Kastoros 72, 18545, Piraeus, \\ Greece; E-Mail: kabouris@desmie.gr \\ * Author to whom correspondence should be addressed; E-Mail: kanellos@mail.ntua.gr; \\ Tel.: +30-210-946-6965; Fax: +30-210-946-6925.
}

Received: 11 August 2009 / Accepted: 5 November 2009 / Published: 9 November 2009

\begin{abstract}
Large penetration of Renewable Energy Sources (RES) impacts Energy Supply Industry (ESI) in many aspects leading to a fundamental change in electric power systems. It raises a number of technical challenges to the Transmission System Operators (TSOs), Distribution System Operators (DSOs) and Wind Turbine Generators (WTG) constructors. This paper aims to present in a thorough and coherent way the redrawn picture for Energy Systems under these conditions. Topics related to emergent technical challenges, technical solutions required and finally the impact on ESI due to large wind power penetration, are analyzed. Finally, general conclusions are extracted about the ESI current and future state and general directions are recommended.
\end{abstract}

Keywords: wind power; large wind penetration; wind integration; energy supply industry; electric market

\section{Introduction}

One major goal for the ESI in Europe is the large-scale penetration of RES. This achievement and the increase in the share of natural gas against fossil fuels are the most important means towards achieving compliance with the Kyoto Protocol commitments. EU has set ambitious targets, aiming at a $20 \%$ share for renewables in the final energy demand by 2020 [1]. The target set for Greece aims to increase the contribution of RES to the final energy consumption to $18 \%$ by 2020 [2]. Among available 
RES technologies, wind power is expected to contribute the largest part and is expected to have the highest evolution.

Since RES technologies are not yet economically competitive with conventional thermal generation, several European countries exclude RES technologies from competition by giving them priority in dispatch [1]; moreover, in almost all the EU member states, RES are promoted through significant incentives such as fixed feed-in tariffs (for instance Germany, Spain, Denmark, Greece, etc), long term contracts but also, in some countries, subsidies on capital investment. These incentives have been proven very successful and significant wind capacity has been installed in many European countries (especially in Germany, Denmark and Spain). It should be mentioned that other market integration approaches - e.g., full exposure of wind generation to market risks-have been proven to be successful as well [3].

The large wind power penetration impacts the ESI both technically and economically. From a technical point of view, ESI faces a variety of technical problems and challenges such as frequency and voltage regulation, available transmission capacities to accommodate RES plants, power quality issues, monitoring and control by the Energy Management Systems (EMS), operational practices, ancillary services, connection interfaces, etc. These issues are currently under investigation by the TSOs in the EU. Concerning electricity economics, large RES penetration will impact emissions, energy balances and generation mix, electricity markets, etc. [4-6]. RES will change the generation mix against conventional thermal generation (mainly the load following generators) and they will reduce proportionally their market share. This impact may be significant for the new market entrants (mainly natural gas combined cycle generators). In this sense, it seems that the targets for market opening and large-scale RES penetration may conflict each other; therefore, policies and regulations in both conventional and RES sectors are crucial to mutually achieve said goals and should be carefully designed. This paper presents the major topics under investigation concerning the impacts of large-scale wind integration into Electric Power Systems.

\section{Technical Challenges, Improvements and Solutions}

\subsection{Wind power characteristics}

Wind power is moving rapidly from being a marginal technology to a major primary source especially within EU. Table 1 summarizes the existing wind installations at the end of 2008. Large scale wind penetration is very likely to be present in the near future. This evolution faces a number of technical challenges and requires considerable changes in a wide range of activities and procedures followed in the electric power systems.

Most of the arising technical challenges are related to one way or another to the stochastic nature of wind. Power systems have to incorporate for first time a source of high uncertainty, high volatility and low predictability [7]. Also, as a supporting mechanism a "priority to dispatch" privilege is offered to the WFs in the EU. This rule can be hardly applied in case of large penetration and raises additional questions for the future. 
Table 1. Indicative figures for various European countries.

\begin{tabular}{cccccc}
\hline Country & $\begin{array}{c}\text { Fossil Fuels } \\
(\mathbf{M W})\end{array}$ & $\begin{array}{c}\text { Renewables } \\
\text { other than } \\
\text { Hydro }(\mathbf{M W})\end{array}$ & $\begin{array}{c}\text { Hydros } \\
\text { (MW) }\end{array}$ & $\begin{array}{c}\text { Wind } \\
\text { Farms } \\
\text { (MW) }\end{array}$ & $\begin{array}{c}\text { Total } \\
\text { NGC } \\
\text { (MW) }\end{array}$ \\
\hline Germany & 72,300 & 32,400 & 9,700 & 23,100 & 134,700 \\
Spain & 43,000 & 19,308 & 18,972 & 15,584 & 88,828 \\
Denmark & 8,815 & 3,793 & 10 & 3,163 & 12,618 \\
France & 24,718 & 4,283 & 25,932 & 3,278 & 117,653 \\
Italy & 73,020 & 4,274 & 21,125 & 3,315 & 98,419 \\
Portugal & 6,804 & 3,170 & 4,957 & 2,640 & 14,931 \\
Netherlands & 21,702 & 3,036 & 38 & 2,216 & 25,261 \\
Greece & 8,375 & 940 & 3,177 & 791 & 12,492 \\
Sweden & 5,093 & 3,955 & 16,195 & 1,021 & 34,181 \\
Belgium & 8,335 & 1,147 & 1,412 & 352 & 16,719 \\
\hline
\end{tabular}

\subsection{Wind turbine technologies}

Although modern wind turbine generators (WTGs) are significantly improved compared to the older technology (80s and 90s) the fact that there is no control on the primary energy source (wind) creates a number of problems in power system operation. Concerning the mechanical power control WTGs can be classified into stall and pitch controlled; the former represent older technology but still in use while the latter reflect the modern technology. Pitch controlled WTGs present several advantages in power control. Concerning the electrical interface, WTGs can be classified into three main categories:

- Fixed speed WTGs equipped with squirrel cage induction generators directly connected to the grid.

- Variable speed WTGs equipped with doubly fed (round rotor) induction generators (rotor circuits are connected to the grid via a back to back converter while stator windings are directly connected to the grid).

- Variable speed WTGs equipped with synchronous generators connected to the grid through a full power back-to-back converter (employing PMW techniques and IGBTs)

All technologies mentioned above are currently in use. As wind penetration increases there is a trend to use more variable speed machines of the last two categories.

\subsection{Crucial technical issues}

In the next sections a brief analysis of the most important technical issues that must be considered and analyzed, is given. These issues address the main field of future developments and changes needed in the TSOs operation in order to overcome limiting factors and achieve high wind penetration without jeopardizing the system security (which is the major task for the TSOs).

\subsubsection{Load following and frequency regulation}

In power systems the total generation must be continuously adjusted to the load demand and keep frequency variation within the range foreseen by the Grid Codes (e.g., $\pm 20 \mathrm{mHz}$ for the interconnected 
system of ENTSO-E). The stochastic nature of wind and the uncontrollability of wind power makes the adjustment of generation to the load difficult since it is undertaken by thermal and hydro generators (and the interconnection transmission lines, if any). Large wind penetration requires increased regulating power (mainly in the level of secondary regulation), i.e., increased spinning reserves. This requirement is expected to increase the cost of the necessary ancillary services to guarantee secure system operation [8]. Due to the large time constants of WTGs they can hardly contribute to primary regulation but the contribution to the secondary regulation is possible and should be further investigated as a measure to improve security in the case of large penetration [6]. Such regulation strategies are already an option for large WFs in countries with high wind penetration (Germany and Denmark). Technically, it is possible for modern WTGs to adjust their production according to commands by the EMS through fast pitch actions, switched loads or speed regulation for WTGs operating at variable speed. This prospect gives rise to a number of important questions, for instance: who will be establishing and financing data acquisition and remote control facilities as such? who will be paying for the lost production? Who will be refunding the loss if the production margin is lowered before a particular time of operation-resulting in the wind turbine owner being unable to deliver the production offered to the exchange? How will the priority between several wind farms be administrated-whose production is going to be restricted? An alternative for effective frequency regulation would be the increase of peaking power plants and the use of pump storage hydros coordinating with the WFs. Finally, it should be noticed that the "load following" related problems are the most severe ones and constitute the most restricting factor for the large wind power penetration.

\subsubsection{Technical characteristics of thermal power plants}

The existing thermal generators comprise a series of technical characteristics which set limits on wind penetration and are related to the load-following capability of the generation system. The technical minimum of thermal generators is the most restricting factor since the majority of the existing generators are non-flexible steam or combined-cycle generators with high technical minima which may restrict wind penetration, especially during low load periods. Also, the start-up and shut-down times needed are usually quite long (especially for coal-fired units) and do not allow the frequent start/stop. In any case frequent start-up/shut down of thermal plants should be avoided to prevent premature aging of the equipment. The ramp-up and ramp-down rates of thermal generators should be also considered. The restrictions set by thermal generators can be alleviated by increasing the available peaking power as mentioned in previous paragraph.

\subsubsection{Available transfer capacities from/to windy areas}

In windy areas human activity is usually limited and consequently the electrical networks are weak. The connection of WFs to electricity grids requires in many cases the construction of significant new transmission infrastructure (lines, substations) which is not always an easy task due to the continuous increase of public opposition against high voltage installations $[9,10]$. Also, the long construction time needed, especially for new transmission lines (mainly due to the complicated authorization procedures and public opposition), may be a strong barrier to the wind exploitation. Cost issues are also crucial for 
future developments. In that aspect the spatial distribution of WFs is crucial in order to reduce the need for new transmission infrastructure.

Concerning the connection interfaces from the WFs to the system boundaries, they are case specific depending on a number of factors such as the short-circuit ratio at the point of connection, the length of the interface, the voltage level, the WF capacity etc. The respective cost is usually undertaken by the WFs' owners.

\subsubsection{Monitoring and control by Energy Management Systems and Metering}

Although WFs have priority in dispatch, under high wind penetration they should be continuously monitored in real time by the Dispatching Centers. In order to ensure security (especially during low load periods) the TSOs must have the possibility to curtail the wind production, if needed. A bidirectional interface between the EMS and WFs is required. The EMS must have the capability to issue commands to the WFs concerning the maximum power injection allowed according to system status and security rules. Due to the high number of WFs extended telecommunication infrastructure is required, usually of considerable cost. Also, the existing SCADA systems must be expanded to accommodate the increasing volume of real time measurements by the WFs.

The everyday operation in Dispatching Centers will be also affected and new software applications are needed (especially for State Estimation and Unit Commitment). The market procedures and operation should be also seen in the view of future high wind penetration. Generally, the high wind penetration will increase dramatically the volume of data to be processed by the EMS tools. The implementation of local Dispatching Centers in windy areas in a decentralized control mode is an option to be considered in order to reduce both the data processing by the National Dispatching Centers and the system complexity.

\subsubsection{Voltage regulation}

Voltage regulation within the permitted range is also a crucial issue for WFs connected both to HV or MV networks. It is, of course, a local problem affecting both the networks and the smooth operation of the WFs themselves. Especially for WFs equipped with asynchronous generators, mechanically switched capacitors are widely used to support voltage. In extreme cases (off-shore wind farms or long connections) the use of SVCs is recommended. Modern WTGs, especially the ones driven to the network through converters, offer interesting possibilities for voltage regulation and may provide voltage support even to the grid as ancillary service.

In case of areas with high wind penetration, the voltage stability of the windy area may be endangered and related issues must be carefully investigated.

\subsubsection{WFs behavior during contingencies}

Conventional synchronous generators are usually equipped with exciter and voltage controls. Also, energy is stored in the magnetic fields in the machine and particularly in the rotor windings. Therefore, synchronous generators are able to supply high short-circuit currents to the fault location for considerable time. In this way, the voltage dip caused by the fault is reduced. In case that many 
conventional synchronous generators are replaced by WFs, WTGs have to provide voltage support during faults and, to some extent, also during normal operation.

Figure 2. Fault Ride-Through requirements in various TSOs.

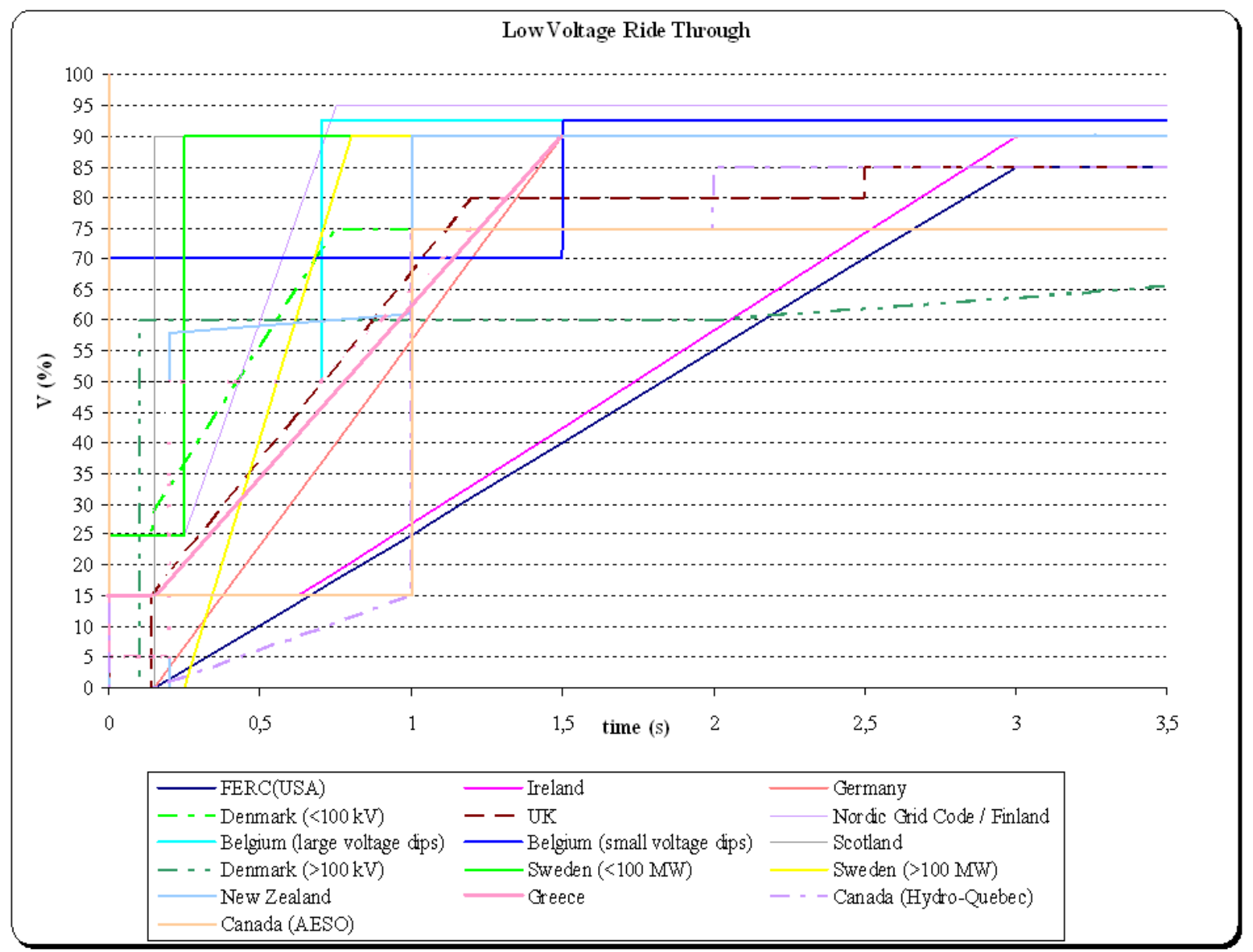

WTGs do not have the ability to provide high short-circuit currents since they are usually not equipped with exciter and voltage controls. During faults that normally happen in the network (short-circuits due to lightning, etc) if voltage drops below a certain level, WTGs trip. As long as Wind power penetration was limited this did not affect the system security significantly and it was even desirable in some cases, but since wind power has become significant, the tripping of WFs in case of a fault could lead to the loss of large amounts of wind power that endangers system security. This leads to the requirement of WFs to stay in synchronism and ride-through the fault (Fault Ride-Through Capability-FRT) [11], i.e., to overcome the voltage variations during transients.

Figure 2 outlines the FRT capability requirements in some EU countries, USA, Canada and New Zealand (information collected from the respective grid codes and [8]). FRT capability is an emerging technology already available by the main WTGs constructors. 


\subsubsection{Impacts on distribution networks}

Wind penetration has also severe effects at the DSO level, i.e., the medium voltage (MV) networks. The main issues concern local effects on power quality and WTGs performance during disturbances. These disturbances are of two types:

- disturbances during the normal system operation (mainly voltage dips) since various factors contribute to voltage fluctuations at the terminals of a wind turbine

- disturbances during faults (short circuits)

More specifically, during the normal system operation the following may appear:

- slow voltage variations due to change in power flows when the WTGs are switched on/off

- fast voltage variations due to changes in wind speed; these variations of the voltage amplitude may cause "flickering" effects

- short duration voltage dips during switching (on/off) of the WTG

- voltage distortion due to harmonics

Also, during network faults, the presence of WTG can lead to:

- malfunctioning of protection equipment since it is designed to operate within strictly 'radial structure' of the MV network.

- increased stress of circuit breakers since the short circuits are additionally fed by the WTGs

- islanding of parts of the grid, fed solely by WTGs, which might cause failure of the end-use equipment but also accidents to the utility's personnel. Such phenomena are more possible in the presence of high capacitance equipment (such as cables)

Therefore, DSOs should reconsider the protection settings of MV networks in the presence of dispersed wind generation.

\subsubsection{Other issues}

Large wind penetration will impact also on the static stability as new oscillation modes are expected. The overall system damping will be also affected. Also, the increase of the short-circuit levels at the point of connection should be taken into consideration.

\subsection{Technological improvements and proposed solutions}

\subsubsection{Improving grid behavior of WTGs}

In order to achieve a more secure operation WTGs should be more grid friendly, i.e., provide ancillary services in terms of frequency and voltage regulation. Concerning voltage, modern WTGs are capable to regulate either their power factor or the output reactive power. It seems that FRT requirement will become a standard practice all over the EU. Concerning frequency control, modern WTGs could provide some reserves by regulating their power output either by using the fast pitch control (some secs) or by temporary storage. Such regulation strategies are under discussion in some EU countries e.g., grid codes of many countries include some of these requirements and are going to be updated to face the increasing challenges. For instance, Greek grid code has been recently updated including FRT requirement and soon many of the above mentioned control requirements are going to be 
included. In any case WFs should participate in the ancillary services markets to motivate WF towards a better system performance.

\subsubsection{Prognosis tools}

Another key issue for the successful integration of WFs is the improvement of wind forecasting. Despite several improvements, wind power forecasting is still fraught with uncertainty. Experience so far has demonstrated that the uncertainty of the present prognostic tools is in the vicinity of $30 \%-40 \%$ for a 36-hour forecast. The accuracy of the prognostic tools should be improved to not more than $10 \%$ to reduce the costs for regulating power to an acceptable level. The use of meteorological data in the wind forecasting tools must become a general practice. It should be mentioned that a lot of progress is being made for wind forecasts closer to real time $[7,12]$.

\subsubsection{Spatial distribution of WFs}

The spatial distribution of WFs is of high importance from many points of view (such as additional transmission infrastructure, wind power volatility, threat of sudden loss of large amounts of wind power etc.). The more the WFs are dispersed within a control area, the less the restrictions in wind power penetration are. Therefore, special care should be given at the planning phase to avoid the concentration of WFs in specific areas.

\section{Impact on Electricity Power Supply}

Apart from the technical impacts large wind penetration will have a severe impact on the ESI economics and structure. Concerning electricity economics significant work has been recently done and useful information can be found in several relative studies [13-15]. These impacts can be classified into the following:

\subsection{Energy balances and electricity cost}

Large wind penetration is expected to change the energy balances in the Electric Power Systems and it will affect the cost of electricity. Wind energy is likely to substitute intermediate and peak load generators, i.e., combined cycles with natural gas and oil burning units. Also, the contribution of imported primary energy is expected to be reduced in EU such increasing the diversity of supply. Since a fixed feed-in tariff is offered to WFs in most of EU countries, the average electricity cost is likely to be increased.

\subsection{Emissions reduction}

Large wind penetration is expected to reduce drastically the greenhouse gases emissions, contributing significantly to the Kyoto goals. Since wind generation replaces mainly peak and load-following units, this contribution must be carefully quantified taking into account realistic estimations about the share of each fuel to the future energy mix. The future emission market structure should consider the external cost of energy and it is crucial for wind development. Green certificates 
introduced recently in some EU countries seem to be an alternative tool to promote RES but it should not be directly associated with the EU electricity trading system mechanisms.

\subsection{Inter-TSOs electricity trading}

Inter-TSOs electricity trading will be also affected by the availability of excess wind power. The evolution in this sector can hardly be predicted since it will depend heavily on the spatial and temporal distribution of wind power throughout Europe. The ensuring of security is a very important issue which must be carefully and mutually investigated by TSOs at least at a regional level. The required Transmission Reliability Margins (TRM) in the interconnectors to ensure security is also an open issue.

\subsection{Reliability and security of supply}

Although wind generation has a random nature, large penetration will increase the overall system reliability of supply. Also, some capacity credits are expected, depending on the spatial and temporal distribution of wind energy, but still difficult to quantify; probabilistic methods should be used for such estimation. Generally, the dispersion of WFs all over a control area will lead to increased capacity credits. Nevertheless, it is doubtful if capacity credits must lead to reduced thermal installed capacity.

\subsection{Impact on generation mix}

As mentioned above, high wind penetration calls for more flexible generation capacity. Thus, more peaking power will be needed. Also, the fuel mix will be affected.

\subsection{Impact on electricity market}

The high volatility of wind will result on higher volatility in System Marginal Price (SMP) and consequently it will affect the economics of thermal generators. This impact, both during peak and medium-load hours, in conjunction with the reduction in their market share due to wind power may be of high importance for the thermal generators' economic viability and especially for the new entrants (mainly combined cycle units and gas turbines) and has to be carefully estimated.

WTGs will normally supply medium and peak load previously supplied by load following and peak-load power plants which are mainly gas turbines and combined cycle plants [10]; since the new units in EU are in most of the cases NGas combined cycles, the reduction of their market share may put economic obstacles to the construction of new power plants. This is one of the most important and crucial issues that regulatory authorities have to resolve in order to avoid delays in the commissioning of new thermal capacity and endanger the generation capacity adequacy within each control area.

Finally, some minor impact is expected in hydrothermal scheduling, maintenance scheduling and transmission pricing.

\section{Conclusions}

Large wind power penetration impacts power systems in many aspects and raises a number of technical challenges to the TSOs, DSOs and WTG constructors. Some major technological 
improvements have been already addressed leading to more grid-friendly WTGs, i.e., provision of ancillary services in terms of frequency and voltage regulation is possible. FRT capability requirements will become a standard practice in order to avoid massive disconnection of WFs in case of system faults. Also, load following-frequency control is a major technical problem in systems with significant wind power production. Concerning frequency control, modern WTGs could provide some reserves by regulating their power output either by using the fast pitch control (some secs) or by temporary storage.

Wind does provide modest amounts of additional installed capacity for planning and reserve purposes and therefore the detailed modeling of WFs is crucial to conduct WFs interconnection and planning studies. Furthermore, the importance of increased flexibility in the non-wind portion of the generating mix is necessary and it could be achieved with more peaking power.

Apart from the technical issues, large wind penetration will have a severe impact on ESI economics and affect also the other market participants. Energy balances and electricity cost, inter-TSOs electricity trading, reliability and security of supply, impact on generation mix and electricity market are important issues that should be carefully analyzed.

Therefore, special care should be given to the careful design of Grid Codes and market regulations by the regulatory bodies in order to ensure the security of supply (in terms of both system security and generation capacity adequacy).

\section{Nomenclature}

$\begin{array}{llll}\text { DSO } & \text { Distribution System Operator } & \text { NGC } & \text { Net Generation Capacity } \\ \text { EMS } & \text { Energy Management System } & \text { PMW } & \text { Pulse Width Modulation } \\ \text { ESI } & \text { Energy Supply Industry } & \text { RES } & \text { Renewable Energy Sources } \\ \text { FRT } & \text { Fault Ride Through } & \text { SVC } & \text { Static Var Compensator } \\ \text { HV } & \text { High Voltage } & \text { TSO } & \text { Transmission System Operator } \\ \text { MV } & \text { Medium Voltage } & \text { WF } & \text { Wind Farm } \\ & & \text { WTG } & \text { Wind Turbine Generator }\end{array}$

\section{References and Notes}

1. Second Strategic Energy Review-An EU Energy Security and Solidarity Action Plan, Europe's current and future energy position Demand-resources, investments. Commission of the European Communities: Brussels, Belgium, 13 November 2008. Available online: http://ec.europa.eu/ climateaction/index_en.htm (accessed on 16 October 2009).

2. Kabouris, J.; Hatziargyriou, N. Wind Power in Greece-Current Situation Future Developments and Prospects. In Proceedings of the IEEE 2006 General Meeting, Montreal, Canada, 18-22 June, 2006.

3. Klessmann, C.; Nabe, C.; Burges, K. Pros and cons of exposing renewables to electricity market risks-A comparison of the market integration approaches in Germany, Spain, and the UK. Energ. Policy 2008, 36, 3646-3661.

4. Erlich, I.; Winter, W.; Dittrich, A. Advanced Grid Requirements for the Integration of Wind Turbines into the German Transmission System. In Proceeedings of the IEEE Power Engineering Society General Meeting, Montreal, Canada, 18-22 June, 2006. 
5. Antoine, J.P.; van Ranst, A.; Stubbe, E.; Derveaux, K.; Janssens, N.; Martinge, H.; Vitet, S.; Jensen, N.M.; Durstewitz, M.; Kabouris, J.; Kanellopoulos, D.V.; Bindner, H. IRENE 2010: Integration of the Renewable Energy in the Electrical Network. In Proceedings of the ALTENER 2000 Conference, Toulouse, France, 23-25 October, 2000.

6. Kanellos, F.; Hatziargyriou, N. Control of variable speed wind turbines in islanded mode of operation. IEEE Transactions on Energy Conversion Journal 2008, 23, 535-543.

7. Smith, J.; Thresher, R.; Zavadil, R.; DeMeo, E.; Piwko, R.; Ernst, B.; Ackermann, T. Mighty wind. IEEE Power and Energy Magazine 2009, 7, 41-51.

8. Holttinen, H.; Lemström, B.; Meibom, P.; Bindner, H.; Orths, A.; Frans van Hulle; Ensslin, C.; Hofmann, L.; Winter, W.; Tuohy, A.; Malley, M.O.; Smith, P.; Pierik, J.; Tande, J.O.; Estanqueiro, A.; Ricardo, J.; Gomez, E.; Söder, L.; Strbac, G.; Shakoor, A.; Smith, J.C.; Parsons, B.; Milligan, M.; Wan, Y. Design and Operation of Power Systems with Large Amounts of Wind Power, Stateof-the-Art Report. VTT Technical Research Center of Finland, October, 2007. Available online: http://www.ieawind.org (accessed on 16 October 2009).

9. Kabouris, J.; Koronides, A.; Manos, G.N.; Papaioannou, A. Development of Network Infrastructure for Bulk Wind Power Injection in Greece. In Proceedings of the EWEA Special Conference "Wind Power for the 21th Century", Kassel, Germany, 25-27 September, 2000.

10. Energy Management Planning for the Integration of Wind Energy into the Grid in Germany, Onshore and Offshore by 2020. Deutsche Energie-Agentur GmbH (DENA): Cologne, Germany, 24 February, 2005. Available online: http://www.offshore-wind.de/page/index.php?id=2605\&L=1 (accessed on 16 October 2009).

11. Buchholz, B.M.; Styczynski, Z.A.; Winter, W. Dynamic Simulation of Renewable Energy Sources and Requirements on Fault Ride through Behavior. In Proceedings of the IEEE Power Engineering Society General Meeting, Montreal, Canada, 18-22 June, 2006.

12. Jursa, R.; Rohrig, K. Short-term wind power forecasting using evolutionary algorithms for the automated specification of artificial intelligence models. Int. J. Forecasting 2008, 24, 694-709. Available online: http://www.iset.uni-kassel.de/pls/w3isetdad/www_iset_new.main_page (accessed on 16 October 2009).

13. Integrating wind-Developing Europe's power market for the large-scale integration of wind power. Tradewind, February, 2009. Available online: http://www.trade-wind.eu (accessed on 16 October 2009).

14. Rüdiger, B.; Christoph, W. Distribution of the Integration Costs of Wind Power. Institute of Energy Economics and the Rational Use of Energy, November 2005. Available online: http://www.wilmar.risoe.dk (accessed on 16 October 2009).

15. Soren, K.; Morthorst, P.E.; Awerbuch, S. The Economics of Wind Energy. EWEA, March 2009. Available online: http://www.ewea.org (accessed on 16 October 2009).

(C) 2009 by the authors; licensee Molecular Diversity Preservation International, Basel, Switzerland. This article is an open-access article distributed under the terms and conditions of the Creative Commons Attribution license (http://creativecommons.org/licenses/by/3.0/). 